\title{
EDUCAÇÃO SANTTÁRIA NUM AMBULATÓRIO DE OBSTETRÍCIA
}

\author{
Sanitary Education in the Obstetrics Clinic \\ Educación Sanitaria en la Clínica de Obstetricia - Espanhol
}

\section{Apresentação}

Fernando Porto ${ }^{1}$

0 artigo intitulado "Educação Sanitária num Ambulatório de Obstetrícia”, publicado no ano de 1950, foi escrito pela enfermeira Feiga Langfeld, formada pela Escola de Enfermagem de São Paulo e responsável pela educação sanitária no Ambulatório de Puericultura da Clínica Obstétrica do Hospital das Clínicas (SP). 0 contexto circunstancial da publicação permeou a regulamentação do ensino da Enfermagem conforme a Lei 775/1949, sendo o primeiro artigo na temática da Enfermagem Obstétrica dentre os dezessete veiculados nos Anais de Enfermagem no período de 1950 a 1954.0 artigo teve como finalidade chamar atenção para o campo da educação preventiva, envolvendo os pacientes de ambulatório, sobretudo de Obstetrícia. Neste sentido, o texto destaca a importância da educação no setor obstétrico com o planejamento do programa para o curso às gestantes, inclusive utilizando algumas adaptações pela Faculdade de Higiene de São Paulo. 0 manuscrito foi direcionado às enfermeiras, mais especificamente às especializadas. A preocupação de Feiga Langfeld para a realização do programa do curso justificava-se em face das dificuldades encontradas pelas pacientes sobre a linguagem médica e da necessidade de educação nos cuidados para o período gravídico-puerperal. Para tanto, a autora pontua às leitoras as características que deveriam ter a enfermeira especializada, objetivando desvelar as dificuldades pessoais das mulheres, para melhor apreensão das orientações, tal como: condição de moradia, consideração aos aspectos sócio-econômicos para possibilitar a promoção de higiene em seu espaço residencial, dentre outros de cunho individual. Já para a realização do programa do curso, voltado para o grupo de mulheres gestantes, destacava a socialização das informações como elemento básico. Para operacionalizar as aulas do curso, as enfermeiras deveriam considerar o nível econômico, social e de instrução do grupo; horário compatível durante o aguardo da consulta médica; e assuntos temáticos de abordagem teórica e prática. Ademais, as aulas deveriam ser repetidas várias vezes durante a semana, em virtude de cada dia ter um grupo diferente de mulheres gestantes, permitindo-as assistir a todas as aulas possíveis durante a gestação. Assim, Langfeld se reporta à experiência semelhante do programa da Escola de Enfermagem da Universidade de Vanderbilit, dos Estados Unidos, denominado "Maternity Nursing", de sucesso na execução. Por fim, a autora apresenta temário das aulas do programa do curso às gestantes, recomenda ambiente adequado, material necessário para o seu desenvolvimento e tempo de aula de cinqüenta minutos, incluindo feedbackdas mulheres. Depreendo deste artigo a preocupação da autora sobre a educação para com a saúde da mulher e do recém-nascido, sinalizando sua vanguarda no campo da Obstetrícia à época, bem como pela difusão daquela prática para a Enfermagem brasileira, que hoje é praticada em muitas instituições de saúde no contexto ambulatorial. 


\section{Presentation}

\section{Fernando Porto'}

The article "Sanitary Education in the Obstetrics Clinic" - Published in 1950 - was written by Nurse Feiga Langfeld graduate of the San Paulo Nursing School and responsible for the Sanitary Education in the Early Childhood Care (Puerperium) Clinic of the Hospital das Clinicas (SP). The circumstantial context of the publication of this article had an influence on the regulations of the teaching of nursing under law 775/1949. It was the first article to mention the obstetrics nurse - among seventeen articles published by The Yearbook of Nursing (Anais da Enfermagem) from 1950 to 1954. The main objective of the present article is to call attention to the fields of prevention and education involving patients from different clinics, especially the ones in the discipline of obstetrics. The article emphasizes the importance of education in obstetrics, and it contains a lesson plan of the program for the a special course for pregnant women - which is currently used, with some adaptations by the Faculty of Hygiene of San Paulo. The manuscript is directed to nurses, and more specifically to specialized nurses. Feiga Langfeld's main concern in creating the lesson plan for this course is justified due to the difficulty of patients to understand medical language, and due to the need of knowledge and information about the care required during pregnancy and early childhood (puerperium). For this purpose, the author teaches her readers the characteristics a specialized nurse should have. She tries to stress women's personal problems in order to obtain a better understanding of orientations such as, living conditions, consideration of social-economic aspects in order to promote hygiene conditions at home, and other personal type issues. On the other hand, in reference to the implementation of the lesson plan for the course, it is created for a group of pregnant women and it emphasizes the socialization of the information as a basic element. In order to create a lesson plan for the classes of the course, the nurses must consider elements such as: social-economic and educational level of the members of the group; that the course's schedule is compatible with the time the patients will wait to be called in for their medical appointments, and thematic subjects of theoretical and practical approach. Besides this, the classes must be repeated several times a week because a different group of pregnant women is present every day. Some of Langfeld's work is based on a similar experience that took place at the nursing program of the Vanderbilt University in the United Sates, called "Maternity Nursing", a program that was carried out with success. And finally, the author presented the programmed class schedule and lesson plan for the course for pregnant women. The author recommends the existence of an adequate environment and the required material for the classes. She suggests a 50 minute class period including feedback from the women in the course. Derived from what it said in this article, the preoccupation of the author with Health Education for women and newborn is highlighted. The work of Nurse Feiga Langfeld was forefront in Obstetrics of her times, and also in the dissemination of this practice within Brazilian Nursing. Nowadays it is practiced in many health institutions (clinics).

\section{Presentación}

Fernando Porto

El articulo titulado "Educación Sanitaria en la Clínica de Obstetricia" - publicado en el año de 1950 - fue escrito por la enfermera Feiga Langfeld - graduada de la Escuela de Enfermería de San Pablo y responsable por la Educación Sanitaria en el Sanatorio de Puericultura de la Clínica Obstétrica del Hospital de las Clínicas (SP). El contexto circunstancial de la edición de esta publicación dejó huella en la reglamentación de la enseñanza de enfermería conforme a la Ley 775/1949. Constituyó el primer artículo que aborda la temática de la enfermería obstétrica, entre diecisiete artículos publicados en los Anales de Enfermería durante el período de 1950 a 1954. El presente artículo tiene como finalidad llamar la atención hacía el campo de la educación preventiva envolviendo pacientes de diferentes clínicas, especialmente del área de obstetricia.

En este sentido, el texto destaca la importancia de la educación en el sector obstétrico, y contiene un planeamiento del programa para el curso especial para gestantes, que es usado inclusive, con algunas adaptaciones, por la Facultad de Higiene de San Paulo. El manuscrito está dirigido a enfermeras, y más específicamente, a las especializadas. La evidente preocupación de Feiga Langfeld por elaborar el programa del curso se justifica ante las dificultades encontradas por los pacientes para entender el lenguaje médico y ante la necesidad de educar e informar sobre los cuidados necesarios durante el periodo embarazo-puerperio. Para lo anterior, la autora señala a las lectoras las características que debería tener una enfermera especializada. Intenta resaltar los problemas personales de las mujeres con el fin de conseguir una mejor comprehensión de orientaciones tales como: condiciones de la vivienda; consideraciones de los aspectos socio-económicos para promover la higiene en el espacio residencial; y otros temas de cuño personal. Por otro lado, en lo que a la realización del programa del curso se refiere, éste está dirigido a un grupo de mujeres gestantes, y hace énfasis en la socialización de las informaciones como elemento básico.

Para la planificación de las clases del curso, las enfermeras deben considerar elementos tales como: el nivel socioeconómico, social y de instrucción de los miembros del grupo; que el horario del curso sea compatible con el tiempo de espera para ser atendido en la consulta médica, y asuntos temáticos de abordaje teórico y práctico. Aunado a lo anterior, las clases deben repetirse varias veces por semana debido a que cada día se presenta un grupo de mujeres gestantes diferentes. De esta forma, Langfeld se basa en una experiencia similar llevada a cabo dentro del programa de enfermería de la Universidad de Vanderbilit - en Estados Unidos - denominado "Maternity Nursing", que fue llevado a cabo con éxito. Por fin, la autora presenta el temario para las clases del programa del curso para gestantes. En éste, recomienda la existencia de un ambiente adecuado; propone el material necesario para el desenvolvimiento del mismo, y sugiere que el tiempo de duración de la clase sea de 50 minutos (incluyendo la retroalimentación (feedback) de las mujeres). De este articulo se desprende la preocupación de la autora con la educación para la salud de la mujer y del recién nacido. Destacándose como una vanguardia en el campo de la obstetricia de la época, así como en la difusión de esta práctica dentro de la enfermería brasileña, que hoy en día es practicada en muchas instituciones de salud (dentro del contexto de sanatorios e clínicas). 


\title{
EDUCAÇÃO SANITÁRIA NUM AMBULATÓRIO DE OBSTETRICIA
}

\author{
FEIGA LANGFELD *
}

Com a evolução da medicina, a "arte" de curar passou da aplicaçăo de conhecimentos empíricos apreendidos na observação léstias, para a utilizacão estudo sistemático das causas das motudo na etiologia e naça de métodos científicos, baseados sobrerismo prolongou-se pràticomia patológica. Essa época de empi-

camente até Pasteur. anteriormente exercia "ciência" de curar, o profissional que contingência de se associar "arte" como um artesão viu-se na nar o campo da medicina, agor desta forma as especializaçóes muito aumentado. Apareceram 0 consultorio médico foi substituido pôs o trabalho de equipe. mente pelo hospital.

O hospital moderno é, pois, na acepção completa do vocábulo, formado de um conjunto de profissionais capazes de dominar o
vasto campo das ciências médicas.

Paralelamente ao desenvolvimento da ciência de curar e mesmo dela derivando, surgiu a medicina preventiva, cuja importância vem aumentando contìnuamente. A higiene, derivada històricamente da medicina, vem se desenvolvendo contìnuamente a ponto de atualmente ser considerada, socialmente em algumas circunstâncias, mais importante que a ciência que lhe deu origem.

\section{IMPORTANCIA DA HIGIENE}

Através da higiene consegue-se melhorar o ambiente em que vive o homem (solo, água, ar, alimento, vestuario, a morádia, etc.) tornando-o saudável para manutenção da saúde. Segundo Afrânio Peixoto, "sua importância revela-se, ainda no cuidado do indivíduo e sua descendência, na prevenção de agravos à saúde

(*) Forma

Aavel pela educaç̃o senitaria de Enfermagem de Sťo Paulo. Enfermelra resnon. cultura da Cifnica Obstétrice do Hospi que Irequentam o Ambulatório de pueri- 
e pelos meios de evitar acidentes, infecçổes, intoxicaçōes, doenças de carência, etc." (1)

Os hospitais do Brasil, em sua maioria, carecem ainda desta finalidade preventiva. Nâo existe na maior parte de nossos hospitais um programa sistemático e organizado no sentido de educar os pacientes dentro de preceitos higiênicos. Já é tempo de se iniciar êsse trabalho tấo necessário, com boa orientação, uma vez que para isso temos elementos tècnicamente aparelhados.

Sabemos que no hospital moderno todo o trabalho é realizado por uma equipe de profissionais e, assim sendo, no setor educativo precisamos tambem que elementos de várias especializações dêm sua contribuição dentro de um programa pre-estabelecido para educação dos pacientes.

Existe no hospital duas maneiras de se assistir aos pacientes; internando os que necessitam de uma tratamento intensivo e de cuidados médicos diàrios ou atendendo os pacientes em ambulatórios, quando nấo há necessidade de uma vigilância diària e constante para o seu tratamento.

A finalidade deste artigo é chamar a atenção para o campo da educação preventiva para os pacientes de ambulatório, sobretudo de obstetrícia. Um ambulatório de obstetrícia é frequentado por pacientes que, até certo ponto, poderiam ser julgadas sãs, mas que precisam consultar periòdicamente a um médico obstetra durante a gestação e no post-partum imediato. A gestante frequenta um ambulatório para que possa ser orientada no que diz respeito aos cuidados que deve ter durante sua gestação e assim prevenir complicações durante a gravidez, no parto e no após parto. Neste ambiente temos um campo ilimitado para a educação, levando-se em conta o baixo nível de instrução das gestantes que frequentam os ambulatórios.

\section{IMPORTÂNCIA DA EDUCAÇÃO NO SETOR OBSTÉTRICO}

I - Dar instruçôes à paciente eliminando, dessa maneira, uma infinidade de defeitos de orientação que nosso povo possue no que se refere à gestação, ao parto e aos cuidados para com $o$ recem-nascido (inclue-se aqui o esclarecimento sôbre muitas superstições existentes).

II - Permitir melhor aproveitamento dos conselhos médi$\cos$, no sentido de compreender e evitar os riseos que possam existir numa gravidez.

(1) Peixoto, Afranio - Higiene - vol. II. Medicina Preventiva - pg. 19. 
III - Educar a gestante e em seguida a mãe, contribuindo assim para maior coeficiente de partos bem sucedidos e recémnascidos sadios.

Num programa de educação, o objeto de todo o trabalho é a paciente e para ela convergem todos os esforesos.

Quais seriam os profissionais envolvidos num programa desta natureza? E' necessário que elementos de vários setores dêm sua contribuição dentro de um programa pré-estabelecido para a: eđucação de gestantes e mães. Êstes profissionais são os médicos, as enfermeiras especializadas, as dietistas e as assistentes sociais.

Se a educaçăo deve ser feita através đe uma equipe, cada profissional tem seu campo específico de ação. Qual seria a atribuição do médico neste programa educaçã̃o? Ao médico, além de examinar a paciente, fazer diagnóstico e dar-lhe tratamento adequado durante a gravidez, compete ainda orientar.

Qual sexia a atribuição da dietista? Tendo estudado os problemas de alimentação, ninguem será mais habilitado que a dietista para dar as aulas atinentes aos assuntos de sua especialidade.

Qual seria a atribuição da assistente social? Levando-se em conta a finalidade do Serviço Social, que é prevenir, curar, ou minorar os males dos indivíduos ou da coletividade no terreno econômico-social, poderenos deduzir que sua ação é valiosa neste setor educativo, uma vez que, um dos maiores entraves para o bom aproveitamento da educação, é o problema econômico social das pacientes. A assistente social com seu preparo técnico especializado está capacitada para compreender a personalidade da paciente, os seus problemas, as suas necessidades e tem meios dentro das possibilidades de nossas organizaçoes para solucionar muitos casos que impedem a paciente de pôr em prática os conhecimentos adquiridos. (2)

Qual seria a atribuição da enfermeira especializada?

A enfermeira especializada deve: $1 .^{\circ}$ receber a orientação do médico e conhecer cada caso de per si. 2. ${ }^{\circ}$ Explicar às pacientes o sentido das instruçóes médicas. $30^{\circ}$ Dar à gestantes em grupos as aulas do programa. $4 .^{\circ}$ Coordenar as atribuições dos. demais elementos, para que o trabalho seja bem desenvolvido.

Como vimos, delimitam-se no setor da educação dois campos preciosos: o da educação individual e o đa educação em grupo. A educação individual imnlica no conhecimento completo da doente, já sob o aspécto clínico, já sob o pisicológico e econômicosocial. A educação individual pode ser feita antes e depois da

(2) Silva, Beatriz Carđoso - Trabalho de conclusẽo do curso, apresentado a. Escols de Serviço Socin1. 
consulta médica, não só para interpretação das ordens médicas, mas também para o esclarecimento dos problemas apresentađos no momento pela paciente.

Quais as dificuldades maiores encontradas pela paciente $\mathrm{e}$ que necessitam educaçã̃o individual?

De maneira geral temos:

1 - Má compreensão das ordens médicas, nesse caso compete à enfermeira esclarecer à paciente todos os pontos que lhe são obscuros.

2 - Dificuldades para a execução de tais pontos. Aquí é preciso averiguar se está em jogo o fator econômico ou pessoal. No primeiro caso, entra a colaboração da assistente social, que, com seu método de pesquisa, vai conhecer a verdadeira situação econômica social da paciente, e tomar as medidas adequadas afim de que o caso tenha a melhor solução. Nas dificuldades pessoais, é preciso que a enfermeira entre em contacto com cada paciente, afim de que possa ter dados para uma boa orientação. Êsses dados seriam: o local da residêneia, o número de pessoas que dormem no quarto, as possibilidade de se promover higiene naquele ambiente. etc.

\section{EDUCAÇÃO EM GRUPO}

Esta parte do programa é de grande importância pois, através dela conseguem-se resultados que não poderiam ser obtidos sòmente pela educaçăo individual. o grupo é o instrumento ideal para suscitar discussóes e com as discussões obteremos material de interesse imediato para o desenvolvimento de preleções futuras, além de permitir que todos tomem conhecimento dos problemas de cada um e das medidas que devem ser adotadas para sua resolução.

\section{PLANIFICAÇÃO DE UM PROGRAMA}

Quando se organiza um programa de aulas para grupo de gestantes, devemos considerar:

1 - O nivel econômico, social e de instrução do grupo.

2 - As possibilidades de comparecimento às aulas, naturalmente, aproveitar-se-á o dia e o tempo em que as pacientes aguardam consulta médica. 


\section{EDUCACÃO SANITARIA EM AMBULATORIO DE OBSTETRICIA}

3 - Distribuição đos assuntos de aula entre os elementos profissionais capazes de ensinar as pacientes com maior clareza. A elaboração do programa deve compreender aulas teóricas e demonstraçôes práticas. Programa como êste tem sido levado a efeito, em muitas maternidades dos Estados Unidos da América do Norte. Temos, por exemplo, o programa da "Maternity Nursing" da Escola de Enfermagem da Universidade de Vanderbilt, um programa que traduzimos e adaptamos. (3) As reuniöes para as aulas poderão ser efetuadas nos dias de consultas no ambulatório. A mesma aula deve ser repetida várias vezes na semana, levando-se em conta que, em cada dia da semana, haverá um grupo. novo para consulta. Poderemos controlar a frequência, organizando um livro no qual serão anotados os nomes das frequentadoras e as aulas assistidas para termos a orientação da sequência: dos pontos do programa. Dêsse modo, cada paciente terá a oportunidade de assistir a todas as aulas durante sua gestação. Poderse-á dar um certificado a cada mãe que assistir a um número. determinado de aulas, o que poderá ser motivo de estímulo e: entusiasmo pelo curso.

\section{PROGRAMA DO CURSO Às GESTANTES (4)}

I - Uma nova vida começa. Como nasce o bebê e como sedesenvolve dentro do corpo da mäe. Importância do bom cuidado pré-natal.

II - Higiene pessoal na gravidêz. Cuidados da gestante, durante a gravidêz.

III - Alimentação para a família e para a gestante. Planos.

IV - A roupa para a gestante antes do nascimento do bebê.

V - Artigos necessários para o bebê. Sono. Limpeza e oquarto do bebê.

VI - As roupas đo bebê. Como vestí-lo.

VII - Parto no Hospital. O que fazer quando o parto começa. O que levar para o hospital.

VIII - Previsốes do hospital para a saúde, confôrto e segurança da mãe e do bebê durante os primeiros dias depois do nascimento. Regras do bom cuidado post. Natal

IX - Banho do bebê (demonstrações).

$\mathrm{X}$ - $\mathrm{O}$ cuidado da mãe e do bebê depois de deixarem o hospital. Importância da alimentação num horário regular.

\footnotetext{
(3) Programs do "Mothers Club" aa Vanderbilt University Hospltal Maternity

(4) Defe programa com algumas variantes es segutdo na Faculdade de Higiene de'Sáde Póblica de Sâ Paulo.
} 
Deve-se ter para o curso uma sala apropriada ou adaptada para êste fim, com todo o material ilustrativo: cartazes vários," sôbre cuidados higiênicos e alimentares, e material de demonstração como: bandeja com pertences para o banho do bebê e demais apetrechos para o banho; um berço feito de caixote ou cesto para com êle se demonstrar as possibilidades de improvisação e ainda material para o preparo do leite artificial.

As aulas devem ser dadas no tempo máximo de 40 minutos para que as pacientes não se cansem, incluindo-se neste tempo 10 minutos de arguição e palestra com as pacientes.

Sugere-se que haja uma enfermeira exclusivamente encarregada dêste trabalho, dada a sobrecarga a que uma enfermeira está sujeita nas suas atribuiçōes habituais e dadas as qualidades especificas que deve possuir para executar êsse tipo de atividade.

A nosso ver, as qualidades desejáveis a uma enfermeira encarregada da parte educacional de uma ambulatório seriam: capacidade didática, ser simples e accessível, de maneira a não criar inibiçóes nas pacientes, dedicação e interesse pela atividade, procurando aumentar seus conhecimentos de maneira a poder resolver com segurança cada vez maior, os problemas surgidos.

As vantagens que decorrem da execução de um programa educacional em um ambulatório de obstetrícia, são de grande proveito para o paciente e hospital. 
